\title{
REVIEW
}

\section{Navigational Bronchoscopy for Early Lung Cancer: A Road to Therapy}

\author{
Kashif Ali Khan · Pietro Nardelli · Alex Jaeger · Conor O'Shea • \\ Padraig Cantillon-Murphy · Marcus P. Kennedy
}

Received: January 11, 2016 / Published online: March 22, 2016

(C) The Author(s) 2016. This article is published with open access at Springerlink.com

\begin{abstract}
Peripheral lung nodules remain challenging for accurate localization and diagnosis. Once identified, there are many strategies for diagnosis with heterogeneous risk benefit analysis. Traditional strategies such as conventional bronchoscopy have poor performance in locating and acquiring the required tissue. Similarly, while computerized-assisted transthoracic needle biopsy is currently the favored diagnostic procedure, it is associated with complications such as pneumothorax and hemorrhage. Video-assisted thoracoscopic and open surgical biopsies are invasive, require general anesthesia and are therefore not a first-line approach. New
\end{abstract}

Enhanced content To view enhanced content for this article go to http://www.medengine.com/Redeem/F784 F0603D6CF572.

K. A. Khan $(\bowtie) \cdot$ M. P. Kennedy

Department of Interventional Pulmonary Medicine,

Cork University Hospital/University College Cork,

Cork, Ireland

e-mail: drkhan95@hotmail.com

P. Nardelli · A. Jaeger · C. O'Shea ·

P. Cantillon-Murphy

School of Engineering, University College Cork,

Cork, Ireland techniques such as ultrathin bronchoscopy and image-based guidance technologies are evolving to improve the diagnosis of peripheral lung lesions. Virtual bronchoscopy and electromagnetic navigation systems are novel technologies based on assisted-computerized tomography images that guide the bronchoscopist toward the target peripheral lesion. This article provides a comprehensive review of these emerging technologies.

Keywords: Bronchoscopy; Computerized tomography (CT); Electromagnetic navigation; Image guidance; Lung cancer; Peripheral lung lesion; Respiratory

\section{INTRODUCTION}

Lung cancer is the most common cause of cancer-related death in the USA and worldwide accounting for more cancer-related deaths compared to colon, breast and prostate combined [1, 2]. Despite advances in diagnostic approaches, staging and treatment, the overall 5-year survival for lung cancer has not significantly changed and is estimated to be 
around $16 \%$ [3]. This is despite the fact that early-detected lung cancer is curable in patients with good performance status. However, the lack of early detection and accurate localization of lesion for tissue acquisition remains one of the biggest challenges in lung cancer management. Indeed, a recently published multicenter early detection screening trial performed using low-dose computerized tomography (CT) has shown the benefit of early detection in a selected high-risk cohort of patients [4]. Screening with low-dose CT results in a relative reduction of $20 \%$ mortality; however, $96.4 \%$ of nodules detected are false positive and $90.4 \%$ of those required further diagnostic investigations [4]. Choosing the most appropriate biopsy technique for suspected peripheral nodule can be a challenging clinical risk-benefit decision and factors such as tumor size, location, patient co-morbidities including emphysematous changes around the lesion, respiratory function and the pre-test probability of malignancy must be taken into account [5-7].

Newer technologies, such as radial probe endobronchial ultrasound (Rp-EBUS), have the advantage of locating the peripheral suspicious lung lesion for cancer; however, they require the operator to have experience, special training, and memory of the three-dimensional tracheobronchial tree. Rp-EBUS does not provide guidance to the bronchoscopist for choosing the correct airway to get to the target lesions; therefore, the overall diagnostic yield is low and operator dependent without the assistance of navigation. Recent advances in navigation systems, based on CT images and devices used to track the position of the bronchoscope, have improved the success rate for the diagnosis of peripheral lung lesion and are recommended in the American College of Chest Physicians (ACCP) guidelines where equipment and expertise are available [7]. As these procedures are performed in a standard bronchoscopic suite, in combination with endobronchial ultrasound-guided transbronchial needle aspiration (EBUS-TBNA) of mediastinal lymph nodes, they may provide a route to complete bronchoscopic thoracic diagnosis and staging of lung cancer during a procedure. Thus in a patient with a peripheral lung nodule and adenopathy, if EBUS-TBNA of mediastinal nodes were negative for cancer, under the same anesthesia the peripheral lesion could be biopsied. However more importantly, these techniques may provide a platform for safer localized therapy for lung cancer. The aim of this paper is to provide an overview of the recent navigation technologies and their clinical applications. This article is based on previously conducted studies and does not involve any new studies of human or animal subjects performed by any of the authors.

\section{VIRTUAL BRONCHOSCOPY AND VIRTUAL BRONCHOSCOPY NAVIGATION}

While computerized-assisted transthoracic needle aspiration is one of the most frequently performed procedures, with the diagnostic yield approaching $65-90 \%$, it is limited due to its complications, in particular pneumothorax, requiring chest tube placement and hospitalization in almost half of the patients in some studies [8-10] (Table 1). Another method, video-assisted thoracic surgery (VATS), is considered a more invasive procedure for the diagnosis of peripheral pulmonary lesion and has its own constraints in terms of cost, duration of the procedure, localization of small nodules during the procedure, single lung ventilation and 
Table 1 A comparison of the multiple modalities available for peripheral pulmonary nodule biopsy

\begin{tabular}{|c|c|c|c|c|c|c|c|}
\hline Characteristics & CT-TTNA & $\begin{array}{l}\text { Standard } \\
\text { bronchoscopy }\end{array}$ & EBUS-TBNA & $\begin{array}{l}\text { Radial } \\
\text { probe } \\
\text { EBUS }\end{array}$ & VB & EMN & BTPNA \\
\hline Image guidance & $\mathrm{CT}$ & Fluoroscopy & $\begin{array}{l}\text { Endoscopic } \\
\text { ultrasound }\end{array}$ & $\begin{array}{l}\text { Endoscopic } \\
\text { ultrasound }\end{array}$ & $\begin{array}{l}\text { Virtual } \\
\text { pathway }\end{array}$ & $\begin{array}{l}\text { Electromagnetic } \\
\text { navigation }\end{array}$ & $\begin{array}{l}\text { Virtual } \\
\text { pathway }\end{array}$ \\
\hline Lung nodules & Yes & Rarely & $\begin{array}{l}\text { Peribronchial } \\
\text { mass }\end{array}$ & $\begin{array}{l}\text { Lung } \\
\text { nodules }\end{array}$ & Yes & Yes & Yes \\
\hline $\begin{array}{l}\text { Mediastinal } \\
\text { lymph nodes }\end{array}$ & $\mathrm{No}^{\mathrm{a}}$ & TBNA & Yes & $\mathrm{No}^{\mathrm{b}}$ & No & Yes & No \\
\hline $\begin{array}{c}\text { Sensitivity for } \\
\text { malignant } \\
\text { lung nodule }\end{array}$ & $65-90 \%$ & $20-84 \%$ & Unknown & $70 \%$ & $44-79 \%$ & $70 \%$ & Unknown \\
\hline Pneumothorax & $15-43 \%$ & Rare & Rare & $1 \%$ & $1.5 \%$ & $1.5-3.5 \%$ & Unknown \\
\hline Cost & $+^{\mathrm{c}}$ & + & $++^{\mathrm{c}}$ & ++ & ++ & $+++^{\mathrm{c}}$ & Unknown \\
\hline
\end{tabular}

BTPNA bronchoscopic, CT-TTNA computerized tomography-guided transthoracic needle aspiration, EBUS endobronchial ultrasound, $E N B$ electromagnetic navigation, TBNA transbronchial needle aspiration, $V B$ virtual bronchoscopy, TPNA transparenchymal nodule access

${ }^{a}$ CT-TTNA is used to access the anterior mediastinal nodes

b A larger radial probe EBUS device is used rarely to guide TBNA of mediastinal nodes; however, it has been replaced by the dedicated EBUS-TBNA scope

${ }^{c}$ CT-TTNA and standard bronchoscopy are common practice.,+++ and +++ are based on initial outlay and cost of consumables. + is an indicative sign and reflects basic cost, ++ is higher and +++ reflects expensive and additional cost

requirement of general anesthesia [11]. It is not a practical approach for all patients with lung nodules suspicious for cancer.

Although routine flexible bronchoscopy has been a conventional method to evaluate peripheral lung nodule, it is of limited diagnostic value [12] with the diagnostic yield ranging from $20 \%$ to $84 \%$ [13-15]. Success is further compromised if the lesion is smaller than $2 \mathrm{~cm}$ [15], due to the inability to go beyond the subsegmental level and to steer endobronchial accessories directly into the lesion.

Therefore to investigate and establish the diagnosis of suspicious lung lesions, particularly located beyond the subsegmental level in the lung, there is a need for a safer and more accurate procedure, not only for early diagnosis of the lesion with less complications, but also to avoid unnecessary delay and surgical procedures. Virtual bronchoscopy is one such CT image-based technique that helps bronchoscopists to get to the lesion. It also forms the basis of novel navigation bronchoscopy technology.

Virtual bronchoscopy (VB) is a computer-guided bronchoscopy simulation facilitated by pre-procedural CT imaging. It provides the same view, angles and zoom settings as real-time bronchoscopy. It is the name given to the indirect and augmented reality visualization of the tracheobronchial tree from the spatial information derived from CT images. A key factor that determines airway 
segmentation and accessible number of branches of the tracheobronchial tree is the quality of the CT image. A good quality CT provides detailed airway segmentation.

Airway segmentation is the process of:

1. automated identification of the airways on each CT slice, with their walls outlined using "threshold" values, so that the computer can recognize the outlined region as airway;

2. the extraction of the identified and the structures outlined from the rest of the image using labels;

3. stacking together of these labeled images as a 3D airway tree.

Specific CT construction image parameters that provide good quality 3D volume, and subsequently detailed airway segmentation and virtual bronchoscopy, are under investigation and development [16]. However, a CT that is appropriately collimated to thin slices $(0.5-1 \mathrm{~mm})$ and overlaps the image reconstruction is generally used to prepare the VB images down to the peripheral bronchi [16, 17].

There are two VB systems commercially available: the BF-NAVI system (Cybernet System Inc., Tokyo, Japan) and the LungPoint $^{\circledR} \quad$ system (Broncus Medical, Mountain View, CA, USA) (Fig. 1). The BF-NAVI has been more extensively studied in humans, whereas LungPoint has limited data in pre-human studies $[18,19]$. The efficacy of VB is variable in the diagnosis of peripheral lung lesions. It has been reported in the range of $44.4-78.9 \%$ [20]. When combined with fluoroscopy, VB has not shown improvement in diagnostic yield with an experienced bronchoscopist [21]. However, when used with radial probe EBUS for peripheral lesions exhibiting involved bronchi on $\mathrm{CT}, \mathrm{VB}$ has been shown to improve the diagnostic yield to $94.4 \%$ vs. $77.8 \%[22,23]$.

The main advantage of this system over electromagnetic navigation and radial probe ultrasound is the lower cost including consumables. Furthermore, during the procedure, virtual bronchoscopy provides information of the airways, where video display of the tracheobronchial tree is not available in real time due to either blood, mucus or airway swelling. The main disadvantage of virtual bronchoscopy is its limitation to guide distal smaller airways, lack of real-time position and characterization of lesions. In cases where the bronchoscope does not reach the lesion, there is no additional guidance. It does not facilitate any remote steering or directional guidance from the operator and essentially matches the virtual model to the airway's actual model without any suitability for tool guidance. Therefore, it is frequently combined with additional guidance modality such as electromagnetic navigation that allows steerable diagnostic tools to improve its efficacy.

\section{ELECTROMAGNETIC NAVIGATION BRONCHOSCOPY}

Electromagnetic navigation bronchoscopy (ENB) is an image-based novel technology that includes the use of virtual bronchoscopy and facilitates approaching peripheral lung lesions and mediastinal lymph nodes, which are difficult to access with conventional bronchoscopy. The components of the ENB are (Fig. 2):

- electromagnetic emitter and tracking board,

- sensor (locatable guide wire),

- EWC (extended working channel),

- CT lung model, and 


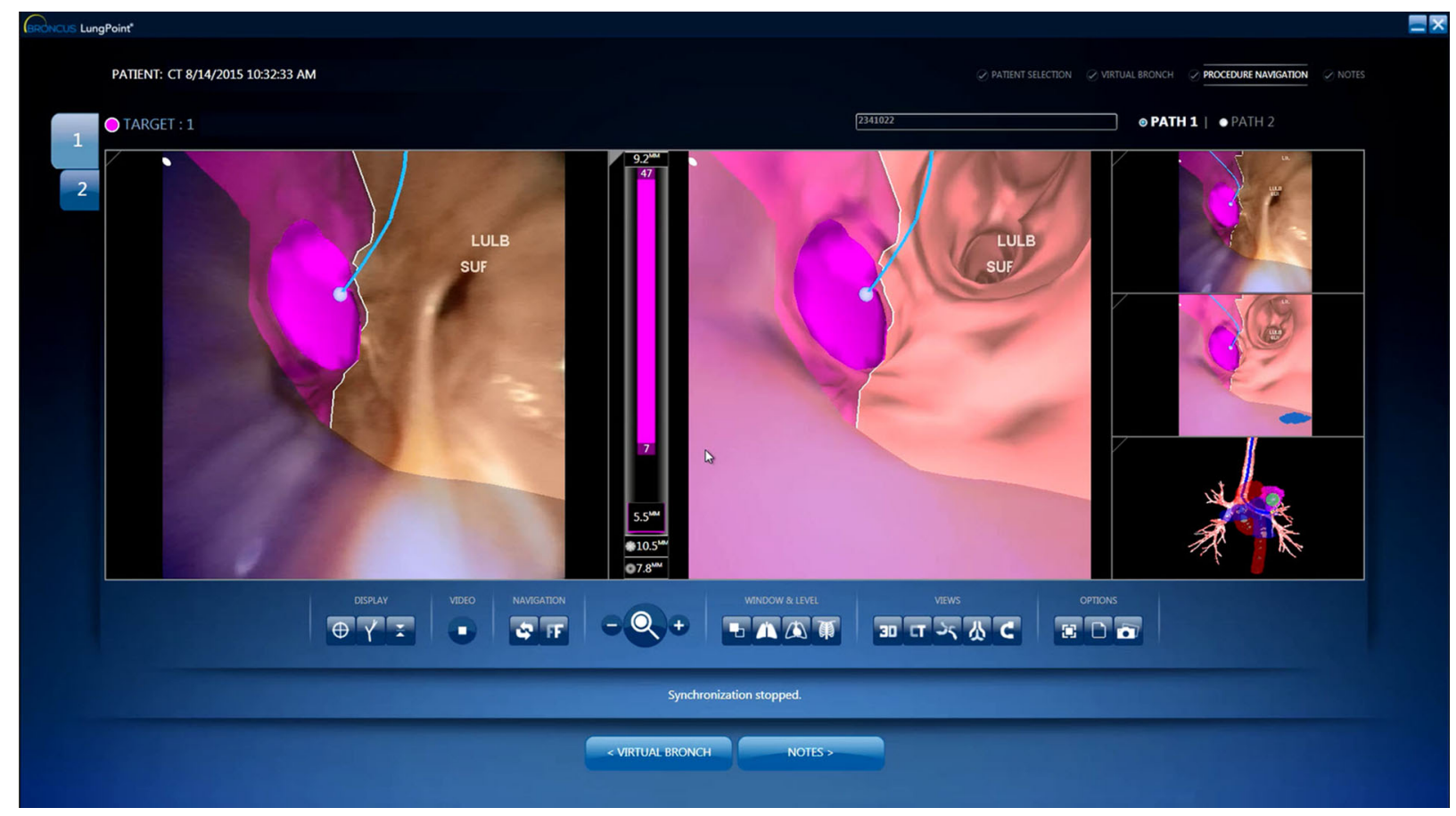

Fig. 1 Virtual bronchoscopy navigation system. Real-time guidance with LungPoint ${ }^{\circledR}$ Virtual Bronchoscopic Navigation simultaneously shows the virtual views and the navigation path. The dark pink area is the target

- Computer-based software.

The electromagnetic emitter and tracking board emits electromagnetic waves that are not affected by air or tissue due to their low frequency $(<30 \mathrm{kHz})$. This creates a magnetic field around the thorax of the patient, in which a sensor position can be tracked with a high degree of accuracy $(<1 \mathrm{~mm})$ [24]. The size of the emitter board and the emitted magnetic field covers the region of interest (thorax, in case of bronchoscopy). The magnetic field can be affected by metal or ferromagnetic interference in the area, which reduces the accuracy; therefore, it needs to be optimally calibrated to the surroundings before clinical use. In commercially available systems, the sensor is usually located at the tip of a flexible cable called a locatable guide (LG) that is covered in a sheath and passed through the working channel selected as target 1 . The blue line indicates the navigation path leading toward the target (courtesy Broncus LungPoint ${ }^{\circledR}$ )

of the scope (EWC). The sensor transfers the information regarding its position, planes and orientation to a computer-based software. The software displays windows of multiple plans on the screen to help navigation toward the target lesion (Fig. 3). Information on location, orientation and movement is superimposed on a previously acquired CT scan and displayed on a monitor in real time [25].

\section{Procedure}

The procedure is performed in several steps: planning, registration and the navigational bronchoscopy. Planning involves the import of CT images in the software on a storage device or through network, in the Digital Imaging and Communications in Medicine (DICOM) format. The CT images are processed through software 




Fig. 2 Our electromagnetic navigation system [16, 25, 33, 34]. In our live porcine model, the essential components of an Electromagnetic Navigation System are labeled. a In a similar manner to Fig. 1, using preloaded data from a standard CT thorax, computer-based software provides virtual bronchoscopy images in a multiplanar working window (monitor screen). b An electromagnetic emitter and tracking board (green) creates a magnetic field around the chest (blue). $\mathbf{c}$ This allows tracking of a sensor probe in

that presents the CT image in axial, coronal and sagittal cuts and also provides VB images and the target lesions (lung nodule, mediastinal lymph nodes). The target lesion or lesions are marked by the bronchoscopist to plan the best possible pathway by identifying the bronchial bifurcation leading toward the target and placing the anatomical waypoints. The virtual environment depends on the quality of the CT images; therefore, it is important to plan the procedure in greater detail. If the particular format is unavailable as per manufacturer's recommendations, then a repeat CT scan is required to utilize the software and technology. the extended working channel of a standard bronchoscope. Coupling of this system to the virtual bronchoscopic images guides movement of the probe to the target lesion. The placement of our probe in a flexible catheter allows passage of standard bronchoscopic instruments and biopsy. d Our research includes the investigation of automation and remote control of our sensor probe and catheter. $E W C$ extended working channel

Once the appropriate pathway is established, the bronchoscopist confirms it by running it in VB and 3D re-constructed tracheobronchial anatomical model, simultaneously following it in the integrated CT images. The planning data can be transferred onto a storage device such as a USB and/or to the computer based in the bronchoscopy room. Before performing the procedure, CT images and both the virtual and real patient environment are aligned and integrated to improve the accuracy of navigation in a process called image to patient "Registration". Registration accuracy is measured as the average fiducial target 

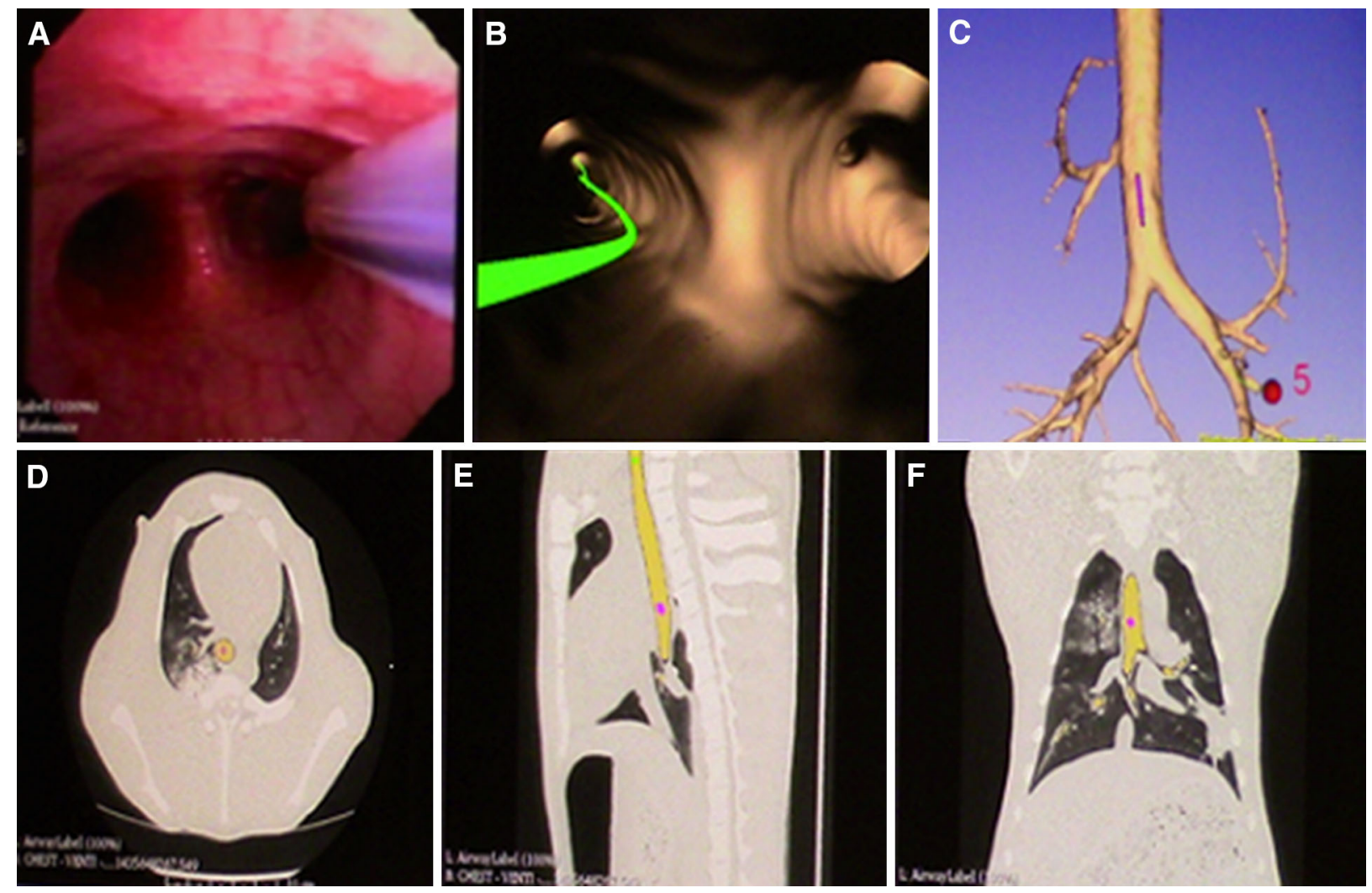

Fig. 3 Our electromagnetic navigation system multiplanar window. a Real-time bronchoscopy image and the sensor probe. b Virtual bronchoscopy image and the navigation pathway (green line) toward the target lesion. c 3D

registration error (AFTRE) and should be $<5 \mathrm{~mm}$. Registration should be repeated in cases where it is $>5 \mathrm{~mm}$, as it signifies unacceptable divergence between the CT and patient anatomy [26, 27]. Navigation is then performed by inserting the sensor probe (covered usually with an external guide sheath) through the working channel of the bronchoscope, thus forming an EWC. From the procedure screen, the operator can choose from multiple available views at different stages of the procedure to fit the purpose of accurate navigation of the target lesion. The position of the probe is simultaneously displayed in all viewpoints. Once the target is located, the EWC is locked on the bronchoscope; the flexible probe can be removed leaving behind tracheobronchial anatomical model. d, e, f Axial, sagittal and coronal images displaying the sensor location in the computerized tomography images [25]

the sheath at the same location for tissue sampling. Various instruments can be introduced through the guide sheath including biopsy forceps, needle and brush. This technology can also guide fiducial placement for targeted therapy [28-30].

There are two commercially available systems that provide ENB: i-Logic ${ }^{\mathrm{TM}}$ (Covedien, Minneapolis, USA) and SPiNDrive ${ }^{\circledR}$ (Veran Medical Technologies, Inc., St Louis, MO, USA). The major differences between the two are the electromagnetic emitter board and the sensor device. The electromagnetic emitter board used in the i-Logic system is placed underneath the patient, whereas in SPiNDrive the field is projected from above the patients. 
The Sensor device in the i-Logic system (LG) is as described above: that is, a probe that can be passed through the working channel of the bronchoscope with a catheter guided sheath which comes in various angles $(45,90$ and 180) and offers manual steerability during navigation. In contrast, the SPiNDrive system uses "Always-On Tip Tracked" instruments: tip tracked aspiration needles, forceps and navigation guide wires that enable ultrathin bronchoscopes to be navigated to the peripheral regions of the lung. Veran Medical Technologies' new "View ${ }^{\mathrm{TM}}$ Peripheral Catheter" is "always-on" with embedded electromagnetic sensor in a disposable catheter that provides real-time guidance throughout the entire procedure. It is a phantom bronchoscope catheter with manual steerability. It has a working channel through which standard instrumentation and sampling can be performed and can also accommodate the use of radial EBUS [31, 32]. Another interesting feature of the Veran Medical Technologies is "percutaneous navigational platform" for tissue sampling during interventional radiology procedures, in particular, electromagnetic CT-guided transthoracic biopsies (E-TTNA) with their navigational needles. E-TTNA has the potential to be performed when ENB is non-diagnostic in the same setting using the same CT scan that was performed for ENB procedure planning and performance. However, at present, there are no clinical trials available comparing outcomes to standard CT-guided lung biopsy.

In addition to the above commercially available electromagnetic navigation systems, our group has developed a 3D electromagnetic navigation platform with airway segmentation and virtual bronchoscopy using an open source 3D slicer environment [25] (Figs. 2, 3). The open source visualization software (3D Slicer http:// www.slicer.org) was further developed to create a detailed airway segmentation and virtual bronchoscopy model from acquired CT images in the DICOM format [16]. A newly developed magnetic field emitter board provides tracking of a semiautomatic locatable sensor catheter in the working channel of the bronchoscope with Always-On Tip Tracked sensor and can be steered both manually and automatically with a joystick, for accurate localization of peripheral lung lesion [33].

Our navigational platform has been tested in vitro using the breathing lung model and in vivo using a porcine model $[25,34]$. The system is at a developmental stage; however, it is distinctive due to being an inexpensive and available open source software. Further work is being carried out to improve the registration and accuracy of the navigational system before a pilot study in patients with peripheral lung nodules [25].

\section{Diagnostic Yield}

The first proof of concept trial to determine the practicality, accuracy and safety of real-time ENB in locating artificial peripheral lung lesions was performed in a swine model in 2003 [35]. The registration accuracy expressed by the fiducial target registration error, expressing both the registration quality and the stability of fiducial (registration) points, was $4.5 \mathrm{~mm}$ on average. ENB was able to successfully reach all nine target areas. No adverse effects, such as pneumothorax or internal bleeding, were encountered in any of the animals in this study. Real-time electromagnetic navigation coupled with a pre-procedure CT scan proved to be an accurate technology that can enhance the effectiveness of standard bronchoscopy to assist in reaching peripheral lung lesion. 
Following this, initial human studies were published in 2005 and 2006; again demonstrating that the ENB with CT scan is a feasible and safe method for obtaining biopsies from peripheral lung lesion. [36, 37] The diagnostic yield reported in both studies was 69\%. No device-related adverse events were reported. Another study during the same time period, with a larger patient group, demonstrated a diagnostic yield of $74 \%$. The study also reported additional applicability of sampling mediastinal lymph nodes with the use of ENB. Since then, a number of studies have added evidence to the use of ENB for the accurate diagnosis of peripheral lung lesion. A recent meta-analysis reported the diagnostic range from $49 \%$ to $87.5 \%$ and the pooled diagnostic yield of $64.9 \%$. The diagnostic accuracy was $73.9 \%$, ranging from $59.0 \%$ to 93.1\% [38-40]. A meta-analysis of the selected 16 studies showed that the median diameter was $25 \mathrm{~mm}$ and the median distance to the pleural surface was $11 \mathrm{~mm}$. Thirty-eight percent of nodules were located in the lower lobes. The sensitivity to detect cancer was $71.1 \%$ (95\% CI 64.6-76.8), with a negative predictive value of $52.1 \%$ (95\% CI 43.5-60.6) [40].

\section{Safety Profile of ENB}

The main complication reported is pneumothorax (3.5\%) which is considerably lower than that with TTNA [8] and reflects the superior safety profile of ENB. In few cases, hypoxemia and minor bleeding have been reported; however, no procedure or sedation-related death has been published [26-28]. In a small case series of fiducial placement, ENB is safe and the pneumothorax rate is smaller than with the transcutaneous implantation of fiducial placement [28-30]. The safety of pacemakers and defibrillators in ENB has been studied in a limited number of patients. There were no complications encountered in all 24 successfully concluded procedures. The plausible explanation for these results is that the magnetic field in ENB is much weaker than that used in MRI and therefore does not interfere with pacemakers and implanted cardiac devices. However, the authors concluded that close cardiac monitoring of patients during and post-procedure is recommended [41].

\section{Factors Influencing Yield}

Despite the improved safety profile, the diagnostic yield of ENB remains less than that of TTNA. There are various patient, operator and technological factors that impact the diagnostic yield.

\section{Patient and Operator Factors}

Firstly, related to lesion, the size and location of the lesion influences the effectiveness of ENB. The ENB platform is used for peripheral lung lesions, although, theoretically, it can be used to diagnose any suspicious lesion, located anywhere in the chest. However, it had been shown [42] that an airway going right into the lesion on the CT scan (bronchus sign) makes the lesion accessible to ENB and improves the diagnostic yield (Fig. 4). If there is no bronchus sign, it is still possible to get to the lesion, but the diagnostic yield may not be as good as it would be if there was a bronchus sign. The diagnosis was $79 \%(30 / 38)$ in patients with a bronchus sign on CT imaging, but only in $4 / 13$ (31\%) with no discernible bronchus sign [42]. Moreover, the diagnostic yield is higher (88\%) for a lesion located in the right middle lobe compared to that in the upper or lower lobes [43]. This may be due to the acute angle of the corresponding bronchus in the upper lobes and 


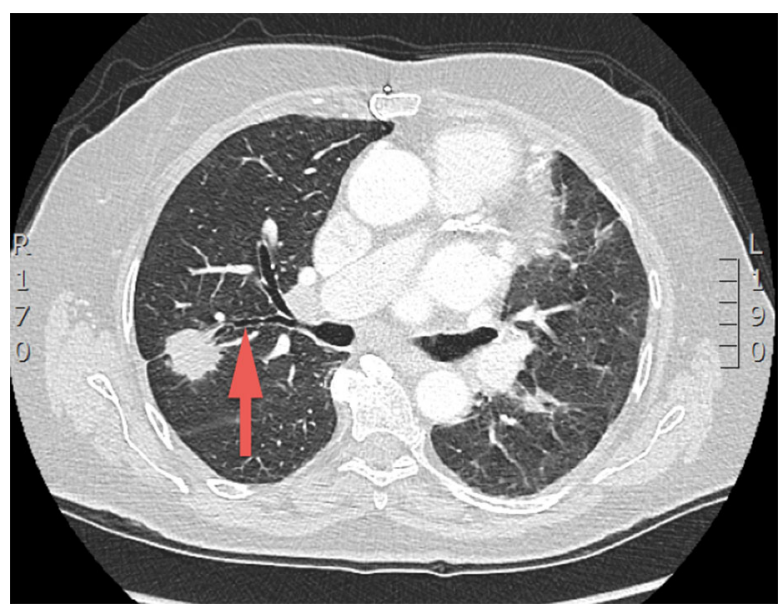

Fig. 4 An 81-year-old lady with bilateral lung nodules. Axial computerized tomography scan shows a positive bronchus sign [red arrow right upper lobe posterior segment (RB2)] related to a mass in her right upper lobe

respiratory movement in the lower lobes [44]. The size of the lesion is also an independent factor that determines the diagnostic yield of the ENB [45]. In a prospective study of 180 consecutive ENB cases, the average lesion diameter was 29.6 vs. $17.6 \mathrm{~mm}$ in the diagnostic and non-diagnostic groups, respectively ( $p=0.001)$ [46].

Surprisingly, the diagnostic yield for peripheral lesions reported in literature, both for the VB and ENB, is not significantly different [20-22, 38-40]. This may be due to lack of clear definition of the peripheral lesion. Pulmonary nodules are defined as $3 \mathrm{~cm}$ or less in size and surrounded by lung parenchyma [5, 47]; however, the definition of peripheral lesion lacks clarity in terms of location from the pleura and whether the lesion is endobronchially at the distal end of the tapering bronchus or completely in the lung parenchyma. The adjacent structures to the lesion or the distance to the pleura may alter the diagnostic yield or complication rate of the procedure. There is a need to clarify and define the peripheral lung lesion due to rapid advancements and comparison of different technologies with variable results [42].

Various other factors that influence the ENB procedures are sedation or general anesthesia, availability of rapid onsite cytology, methods of tissue acquisition and operator experience. Studies differ with regard to yield with conscious vs. general anesthesia [38, 43, 48-50]. However, a recent meta-analysis has shown that the diagnostic yield was better with the use of general anesthesia (69.2\% vs. $57.5 \%$, $p$ 0.02) [40]. The optimal method remains arguable and also depends on local expertise.

Rapid on-site cytology (ROSE) utilizes immediate analysis of the aspirated specimens and provides instant feedback about the specimen quality and diagnosis. The use of ROSE improves the diagnostic yield [40, 51, 52]. The methods of tissue acquisition have also been studied; catheter aspiration compared to traditional practice of forceps biopsy has been shown to have higher diagnostic yield in one study (90\% vs. 50\%) [53]. However, similar to the research of diagnostic tools in radial probe ultrasound, a broad armamentarium for sampling is better once the lesion has been accurately localized. Further research is required with regard to the optimal combination of sampling techniques. The learning curve for ENB is variable and operator dependent. The centers using ENB have demonstrated higher diagnostic yield with improvement in operator experience [54]

\section{Technological Factors}

Not surprisingly, factors that have affected the outcome of the procedure are related to the technology itself, including the AFTRE score. Low AFTRE $<5 \mathrm{~mm}$ indicates less CT to patient divergence and improved registration, and results in better diagnostic accuracy [26, 27]. Various other technological factors that have 
been studied are CT configuration and optimization (reconstruction kernel, radiation dose and slice thickness) for detailed 3D segmentation [16], and the improvement of software to make it user friendly and cost-effective to help clinicians incorporate this technology in their practice.

\section{COMPLEMENTARY TECHNOLOGIES}

\section{Radial Probe EBUS}

Combined modalities can overcome the limitations of individual techniques. A technology that complements ENB is Rp-EBUS, in that ENB provides the guidance lacking in Rp-EBUS. A meta-analysis of Rp-EBUS identified a sensitivity of $73 \%$ with a pneumothorax rate of $1 \%$; however, the selection of patients included in these trials must be taken into account [55]. A subsequent meta-analysis of guided bronchoscopy for the evaluation of the pulmonary nodule for all bronchoscopic modalities identified similar sensitivity for lung cancer and complication rates [56]. Combining ENB and Rp-EBUS, Rp-EBUS may provide better visualization and a further degree of certainty that the target has been reached. Rp-EBUS is performed by placing the tip of a $20 \mathrm{MHz}$ miniature ultrasonic radial probe at the site of the lesion through the working channel of the bronchoscope. A prospective randomized trial has shown an improved diagnostic yield of $88 \%$ with a combination of ENB and Rp-EBUS, in comparison to Rp-EBUS alone (69\%) and ENB alone (59\%) [38]. Both fluoroscopy and ROSE were not used during the study. Moreover, there was significantly less bleeding and the pneumothorax rate was $8 \%$ in the combined ENB/Rp-EBUS procedure [8, 57]. Another trial combining the VB and Rp-EBUS in patients with a positive bronchus sign (Fig. 4) showed that the diagnostic yield in the $\mathrm{VB}$ and non-VB groups was $94.4 \%$ vs. $77.8 \% \quad(p=0.004)$, respectively, particularly high for lesions smaller than $20 \mathrm{~mm} \quad(94.6 \%$ vs. $70.7 \%$; $p=0.006$ ) [22]. A more recent trial suggested that the use of ENB after bronchoscopy, with a combination of Rp-EBUS and VB, led to a small increased yield with an overall yield of $70 \%$ [29]. As before, probe position and lesion size influenced diagnostic yield; the authors suggest using all available modes of sampling when the lesion is reached. In interpreting trials, the expertise of the individual bronchoscopist must be considered and multicenter standardized comparisons would be extremely helpful in clarifying the best diagnostic pathway for peripheral lung nodules.

Additionally, multimodality guidance for bronchoscopic insertion of fiducial markers has been used successfully and safely for accurate external beam stereotactic radiation treatment $[29,30]$. Intratumor localization of fiducial marker placement was superior and the complications including fiducial migration and pneumothorax were less, compared to the percutaneous fiducial lung implantation in the multimodality group $[29,30]$. Overall, combining multimodalities in one procedure are of great interest for the future development of navigational technology.

\section{ULTRATHIN BRONCHOSCOPY}

Ultrathin bronchoscopy (UTB) allows reaching the bronchial tree beyond the conventional bronchoscope. It has an outer diameter of around $3 \mathrm{~mm}$ and the inner working channel diameter of just over $1 \mathrm{~mm}(1.2 \mathrm{~mm})$. This allows deeper access into the endobronchial tree. However, given the thin and malleable 
nature of the scope with small working channel, it may pose difficulty in maneuvering through distal airways. A recent comparative trial for peripheral pulmonary lesion in 310 patients, combining either UTB or thin bronchoscopy (TB) that has external diameter of $4 \mathrm{~mm}$ with EBUS, fluoroscopy and VB has shown higher diagnostic histologic specimens in UTB vs. TB (74\% vs. 59\%; $p=0.044$ ) [58].

\section{BRONCHOSCOPIC TRANSPARENCHYMAL NODULE ACCESS}

One of the limitations of ENB is the challenge of accessing the nodules, which are eccentrically positioned and may not have the airway directly leading to them. To overcome this, a novel navigational approach, bronchoscopic transparenchymal nodule access (BTPNA) has been recently developed [59-61]. In this technique, the nodules are accessed through a transparenchymal "off-road" approach that is not dependent on the need to have an airway leading into the lesion. A computer software-generated tunneled path is created from the bronchial segments through the lung parenchyma directly to the peripheral lung lesions (Fig. 5). A pilot study in a human population has demonstrated the safety and feasibility with adequate sampling and a yield of $83 \% \quad(10 / 12$ lesions) [59]. The mean transparenchymal tunnel size was $47 \mathrm{~mm}$ (10-90 mm), and an average of 3.3 samples was obtained in 12 patients. In this study, Archimedes Virtual Bronchoscopy Navigation System (Broncus Medical, Mountain View, CA, USA) was used in combination with computer-enhanced fused fluoroscopy. The mean fluoroscopy time was 5.5 (range 1.8-11.6) min. There was no pneumothorax or significant complications apart from transient troponin leak. However, in 2 out of 12 patients, the track could not be created [59]. BTPNA relies on the operator experience and the software to ensure that no major vessels are injured while tunneling through the lung parenchyma. Further data to compare BTPNA against the current standard of transthoracic biopsy is warranted.

\section{BRONCHOSCOPIC-GUIDED THERAPY FOR EARLY-STAGE PERIPHERAL LUNG CANCER}

At present, bronchoscopic-guided therapy for lung cancer is limited to debulking of malignant airway obstruction with a number of different techniques including electrocautery [62] and treatment of early in situ cancer for the airways [62]. However, given the access to cancer through novel bronchoscopic techniques, there is a growing interest in bronchoscopic-guided therapy for early lung cancer, such as brachytherapy microwave ablation and radiofrequency ablation.

At present to our knowledge, only one group has presented their experience in a small case series. Delivery of cancer-directed therapy (brachytherapy) by ENB guidance has been demonstrated in the major airways [63, 64]. However, the performance of this technique in comparison to standard therapy is not clear and continues to remain a potential research area.

\section{COST ANALYSIS}

There have been limited cost analysis studies of these techniques. It is widely accepted that CT-TTNA is cost-effective in comparison to VATS biopsy, especially with the availability of positron emission tomography-CT [65]. 


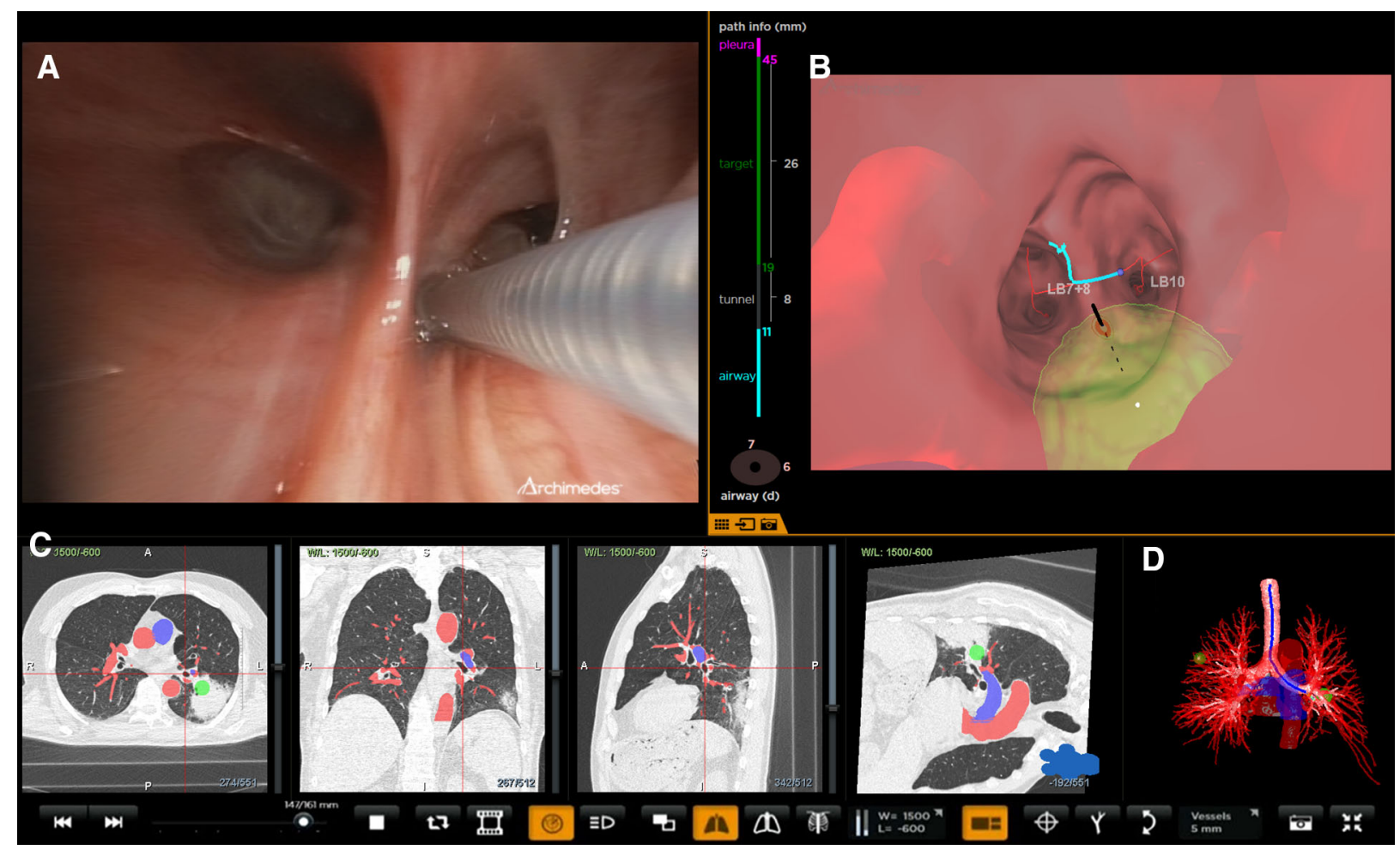

Fig. 5 Bronchoscopic transparenchymal nodule access (courtesy Broncus Archimedes ${ }^{\mathrm{TM}}$ ). a Real-time bronchoscopic image showing catheter at the point of entry. b Real-time virtual image with point of entry overlaid. Green area indicates target lesion. The vertical colored lines on the left of the virtual bronchoscopy indicate

Another study applied a decision tree analysis to compare downstream costs of Rp-EBUS with CT-TTNA [66]. The costs of Rp-EBUS and CT-TTNA appeared to be equivalent (US\$2724 vs. US\$2748), and the authors suggested that specific clinical-radiologic factors such as a positive bronchus sign and location of the lesion influence cost-benefit outcomes. Further evaluation of patient preferences and their influence on cost-effectiveness were recommended. Finally, another study using 100 hypothetical patients created a decision tree with values from the literature to evaluate the clinical consequences and societal costs of a CT-TTNA versus ENB biopsy diagnosis of an SPN [67]. The authors concluded that an ENB with the distance in the airway, tunnel length, size of the target and distance from the pleura. c Computerized tomography projections with target and vessel overlay. Red and blue colors indicate vessels. The target is marked green. d 3D reconstruction of the airway tree

biopsy strategy is associated with decreased pneumothorax rate, but increased costs (by 3719US\$ per case) and increased use of VATS (by 20\%). Combining CT-guided biopsy and ENB with biopsy serially can decrease costs and complications. This, however, is clearly a significant cost increase and needs to be investigated further (Table 1). Ideally, a strategy combining EBUS-TBNA of mediastinal lymph nodes followed by sequential guided biopsy techniques should be compared to separate mediastinal sampling plus CT-TTNA. Improvements in dedicated EBUS scope such as reduction in size and increased angulation will allow bronchoscopists to use this device to biopsy more peripheral lung lesions [68]. 


\section{CONCLUSIONS}

In conclusion, navigational bronchoscopic techniques are an exciting advancement that improves the success in the diagnosis of peripheral lung lesion. They allow a "one-stop shop" of accurate bronchoscopic assessment of both the mediastinum through EBUS or ENB and the peripheral lesion. However, improvements are required in accuracy, and the procedure is currently expensive particularly after adding the expense of obtaining additional thin-slice CT prior to the procedure and the disposable equipment that further drives up the cost.

These techniques appear safe and promising, not only for the diagnosis of peripheral lung lesion, but also holds hopes for therapeutic applications like microwave ablation, radiofrequency ablation and brachytherapy.

\section{ACKNOWLEDGMENTS}

No funding or sponsorship was received for this study or publication of this article. All named authors meet the International Committee of Medical Journal Editors (ICMJE) criteria for authorship for this manuscript, take responsibility for the integrity of the work as a whole and have given final approval for the version to be published.

Disclosures. Kashif Ali Khan, Pietro Nardelli, Alex Jaeger, Conor O'Shea, Padraig Cantillon-Murphy and Marcus P. Kennedy have nothing to disclose.

Compliance with Ethics Guidelines. This article is based on previously conducted studies and does not involve any new studies of human or animal subjects performed by any of the authors.
Open Access. This article is distributed under the terms of the Creative Commons Attribution-NonCommercial 4.0 International License (http://creativecommons.org/licenses/ by-nc/4.0/), which permits any noncommercial use, distribution, and reproduction in any medium, provided you give appropriate credit to the original author(s) and the source, provide a link to the Creative Commons license, and indicate if changes were made.

\section{REFERENCES}

1. Siegel R, Ward E, Brawley O, et al. Cancer statistics, 2011: the impact of eliminating socioeconomic and racial disparities on premature cancer deaths. CA Cancer J Clin. 2011;61(4):212-36.

2. Jemal A, Bray F, Center MM, et al. Global cancer statistics. CA Cancer J Clin. 2011;61(2):69-90.

3. Goldstraw P, Crowley J, Chansky K, et al. The IASLC Lung Cancer Staging Project: proposals for the revision of the TNM stage groupings in the forthcoming (seventh) edition of the TNM classification of malignant tumours. J Thorac Oncol. 2007;2(8):706-14.

4. National Lung Screening Trial Research T, Aberle DR, Adams AM, et al. Reduced lung-cancer mortality with low-dose computed tomographic screening. N Engl J Med. 2011;365(5):395-409.

5. Gould MK, Donington J, Lynch WR, et al. Evaluation of individuals with pulmonary nodules: when is it lung cancer? Diagnosis and management of lung cancer, 3rd ed: American College of Chest Physicians evidence-based clinical practice guidelines. Chest. 2013;143(5 Suppl):e93S-120S.

6. Ost DE, Yeung SC, Tanoue LT, et al. Clinical and organizational factors in the initial evaluation of patients with lung cancer: diagnosis and management of lung cancer, 3rd ed: American College of Chest Physicians evidence-based clinical practice guidelines. Chest. 2013;143(5 Suppl):e121S-41S.

7. Detterbeck FC, Lewis SZ, Diekemper R, et al. Executive Summary: diagnosis and management of lung cancer, 3rd ed: American College of Chest Physicians evidence-based clinical practice guidelines. Chest. 2013;143(5 Suppl):7S-37S. 
8. Khan KA, Zaidi S, Swan N, et al. The use of computerised tomography guided percutaneous fine needle aspiration in the evaluation of solitary pulmonary nodules. Ir Med J. 2012;105(2):50-2.

9. Santambrogio L, Nosotti M, Bellaviti N, et al. CT-guided fine-needle aspiration cytology of solitary pulmonary nodules: a prospective, randomized study of immediate cytologic evaluation. Chest. 1997;112(2):423-5.

10. Laurent F, Michel $P$, Latrabe $V$, et al. Pneumothoraces and chest tube placement after CT-guided transthoracic lung biopsy using a coaxial technique: incidence and risk factors. AJR Am J Roentgenol. 1999;172(4):1049-53.

11. Gould MK, Sanders GD, Barnett PG, et al. Cost-effectiveness of alternative management strategies for patients with solitary pulmonary nodules. Ann Intern Med. 2003;138(9):724-35.

12. Baaklini WA, Reinoso MA, Gorin $\mathrm{AB}$, et al. Diagnostic yield of fiberoptic bronchoscopy in evaluating solitary pulmonary nodules. Chest. 2000;117(4):1049-54.

13. Funakoshi $\mathrm{Y}$, Sawabata N, Takeda $\mathrm{S}$, et al. Bronchoscopically undiagnosed small peripheral lung tumors. Interact CardioVasc Thorac Surg. 2003;2(4):517-20.

14. Shiner RJ, Rosenman J, Katz I, et al. Bronchoscopic evaluation of peripheral lung tumours. Thorax. 1988;43(11):887-9.

15. Schreiber G, McCrory DC. Performance characteristics of different modalities for diagnosis of suspected lung cancer: summary of published evidence. Chest. 2003;123(1 Suppl):115S-28S.

16. Nardelli P, Khan KA, Corvo A, et al. Optimizing parameters of an open-source airway segmentation algorithm using different CT images. Biomed Eng Online. 2015;14:62.

17. Neumann K, Winterer J, Kimmig M, et al. Real-time interactive virtual endoscopy of the tracheo-bronchial system: influence of CT imaging protocols and observer ability. Eur J Radiol. 2000;33(1):50-4.

18. Merritt SA, Gibbs JD, Yu KC, et al. Image-guided bronchoscopy for peripheral lung lesions: a phantom study. Chest. 2008;134(5):1017-26.

19. Dolina MY, Cornish DC, Merritt SA, et al. Interbronchoscopist variability in endobronchial path selection: a simulation study. Chest. 2008;133(4):897-905.
20. Asano F, Eberhardt R, Herth FJ. Virtual bronchoscopic navigation for peripheral pulmonary lesions. Respiration. 2014;88(5):430-40.

21. Tachihara M, Ishida $\mathrm{T}$, Kanazawa $\mathrm{K}$, et al. A virtual bronchoscopic navigation system under X-ray fluoroscopy for transbronchial diagnosis of small peripheral pulmonary lesions. Lung Cancer. 2007;57(3):322-7.

22. Asano F, Shinagawa N, Ishida T, et al. Virtual bronchoscopic navigation improves the diagnostic yield of radial-endobronchial ultrasound for peripheral pulmonary lesions with involved bronchi on CT. Intern Med. 2015;54(9):1021-5.

23. Oshige $M$, Shirakawa T, Nakamura M, et al. Clinical application of virtual bronchoscopic navigation system for peripheral lung lesions. J Bronchol Interv Pulmonol. 2011;18(2):196-202.

24. O'Donoghue K, Cantillon-Murphy P. Low cost super-Nyquist asynchronous demodulation for use in EM tracking systems. IEEE Trans Instrum Measure. 2014;64(2):458-66.

25. Khan KA, Nardelli P, Alex J, O'Shea C, Cantillon-Murphy P, Kennedy MP. The design and validation of a novel semiautomatic lung navigation platform. Thorax. 2015;70(Supple 3):A220.

26. Makris D, Scherpereel A, Leroy S, et al. Electromagnetic navigation diagnostic bronchoscopy for small peripheral lung lesions. Eur Respir J. 2007;29(6):1187-92.

27. Makris D, Gourgoulianis KI. Electromagnetic navigation diagnostic bronchoscopy and transbronchial biopsy. Chest. 2008;133(3):829-30.

28. Hagmeyer L, Priegnitz C, Kocher M et al. Fiducial marker placement via conventional or electromagnetic navigation bronchoscopy (ENB): an interdisciplinary approach to the curative management of lung cancer. Clin Respir J. 2014. doi:10.1111/crj.12214

29. Steinfort DP, Siva S, Kron T, et al. Multimodality guidance for accurate bronchoscopic insertion of fiducial markers. J Thorac Oncol. 2015;10(2):324-30.

30. Schroeder C, Hejal R, Linden PA. Coil spring fiducial markers placed safely using navigation bronchoscopy in inoperable patients allows accurate delivery of CyberKnife stereotactic radiosurgery. J Thorac Cardiovasc Surg. 2010;140(5):1137-42.

31. von Jako RA, Carrino JA, Yonemura KS, et al. Electromagnetic navigation for percutaneous guide-wire insertion: accuracy and efficiency 
compared to conventional fluoroscopic guidance. Neuroimage. 2009;47(Suppl 2):T127-32.

32. http://www.veranmedical.com/view-catheter.html. Accessed 12 Aug 2015.

33. O'Donoghue K, Corvo A, Nardelli P, et al. Evaluation of a novel tracking system in a breathing lung model. Conf Proc IEEE Eng Med Biol Soc. 2014;2014:4046-9.

34. O'Shea C, Khan KA, Kennedy MP, Nardelli P, Cantillon-Murphy P. Evaluation of endoscopically deployed radiopaque tumour models in bronchoscopy. J Bronchol Interv Pulmonol (Manuscript accepted).

35. Schwarz Y, Mehta AC, Ernst A, et al. Electromagnetic navigation during flexible bronchoscopy. Respiration. 2003;70(5):516-22.

36. Schwarz Y, Greif J, Becker HD, et al. Real-time electromagnetic navigation bronchoscopy to peripheral lung lesions using overlaid CT images: the first human study. Chest. 2006;129(4):988-94.

37. Becker HD, Herth F, et al. Bronchoscopic biopsy of peripheral lung lesions under electromagnetic guidance. A pilot study. J Bronchol Interv Pulmonol. 2005;12:9-13.

38. Eberhardt R, Anantham D, Ernst A, et al. Multimodality bronchoscopic diagnosis of peripheral lung lesions: a randomized controlled trial. Am J Respir Crit Care Med. 2007;176(1):36-41.

39. Lamprecht B, Porsch P, Wegleitner B, et al. Electromagnetic navigation bronchoscopy (ENB): Increasing diagnostic yield. Respir Med. 2012;106(5):710-5.

40. Gex G, Pralong JA, Combescure C, et al. Diagnostic yield and safety of electromagnetic navigation bronchoscopy for lung nodules: a systematic review and meta-analysis. Respiration. 2014;87(2):165-76.

41. Khan AY, Berkowitz D, Krimsky WS, et al. Safety of pacemakers and defibrillators in electromagnetic navigation bronchoscopy. Chest. 2013;143(1):75-81.

42. Seijo LM, de Torres JP, Lozano MD, et al. Diagnostic yield of electromagnetic navigation bronchoscopy is highly dependent on the presence of a Bronchus sign on CT imaging: results from a prospective study. Chest. 2010;138(6):1316-21.

43. Eberhardt R, Anantham D, Herth F, et al. Electromagnetic navigation diagnostic bronchoscopy in peripheral lung lesions. Chest. 2007;131(6):1800-5.
44. Chen A, Pastis N, Furukawa B, et al. The effect of respiratory motion on pulmonary nodule location during electromagnetic navigation bronchoscopy. Chest. 2015;147(5):1275-81.

45. Jensen KW, Hsia DW, Seijo LM, et al. Multicenter experience with electromagnetic navigation bronchoscopy for the diagnosis of pulmonary nodules. J Bronchol Interv Pulmonol. 2012;19(3):195-9.

46. Ortega PR, Gutiérrez JG, Juárez ÁOR, Arias HDQ, Rendon JLS, Botella JAB, López-Zalduendo E, Guevara JZ, Salcedo PAS, Ezquibela AC, Ocaña ABA, De Torres Tajes JP, Escario MDL, Maceiras LS, Zulueta JJ. Diagnostic yield and complications associated with electromagnetic navigation bronchoscopy in peripheral lung lesions. Eur Respir J. 2014;44(Suppl 58):S686.

47. Ost D, Fein AM, Feinsilver SH. Clinical practice. The solitary pulmonary nodule. $\mathrm{N}$ Engl J Med. 2003;348(25):2535-42.

48. Brownback KR, Quijano F, Latham $\mathrm{HE}$, et al. Electromagnetic navigational bronchoscopy in the diagnosis of lung lesions. J Bronchol Interv Pulmonol. 2012;19(2):91-7.

49. Pearlstein DP, Quinn CC, Burtis CC, et al. Electromagnetic navigation bronchoscopy performed by thoracic surgeons: one center's early success. Ann Thorac Surg. 2012;93(3):944-9 (discussion 949-50).

50. Bowling MR, Kohan MW, Walker P, et al. The effect of general anesthesia versus intravenous sedation on diagnostic yield and success in electromagnetic navigation bronchoscopy. J Bronchol Interv Pulmonol. 2015;22(1):5-13.

51. Karnak D, Ciledag A, Ceyhan K, et al. Rapid on-site evaluation and low registration error enhance the success of electromagnetic navigation bronchoscopy. Ann Thorac Med. 2013;8(1):28-32.

52. Chenna P, Chen AC. Radial probe endobronchial ultrasound and novel navigation biopsy techniques. Semin Respir Crit Care Med. $2014 ; 35(6): 645-54$.

53. Eberhardt R, Morgan RK, Ernst A, et al. Comparison of suction catheter versus forceps biopsy for sampling of solitary pulmonary nodules guided by electromagnetic navigational bronchoscopy. Respiration. 2010;79(1):54-60.

54. Bansal S, Hale K, Sethi S, Cicenia JC. Electromagnetic navigational bronchoscopy: a learning curve analysis. Chest Meet. 2007;132:514.

55. Steinfort DP, Khor YH, Manser RL, et al. Radial probe endobronchial ultrasound for the diagnosis 
of peripheral lung cancer: systematic review and meta-analysis. Eur Respir J. 2011;37(4):902-10.

56. Memoli JSW, Nietert PJ, Silvestri GA. Meta-analysis of guided bronchoscopy for the evaluation of the pulmonary nodule. Chest. 2012;142(2):385-93.

57. Yeow KM, Su IH, Pan KT, et al. Risk factors of pneumothorax and bleeding: multivariate analysis of 660 CT-guided coaxial cutting needle lung biopsies. Chest. 2004;126(3):748-54.

58. Oki M, Saka H, Ando M, et al. Ultrathin bronchoscopy with multimodal devices for peripheral pulmonary lesions. A randomized trial. Am J Respir Crit Care Med. 2015;192(4):468-76.

59. Herth FJ, Eberhardt R, Sterman D, et al. Bronchoscopic transparenchymal nodule access (BTPNA): first in human trial of a novel procedure for sampling solitary pulmonary nodules. Thorax. 2015;70(4):326-32.

60. Sterman DH, Keast T, Rai L, et al. High yield of bronchoscopic transparenchymal nodule access real-time image-guided sampling in a novel model of small pulmonary nodules in canines. Chest. 2015;147(3):700-7.

61. Silvestri GA, Herth FJ, Keast T, et al. Feasibility and safety of bronchoscopic transparenchymal nodule access in canines: a new real-time image-guided approach to lung lesions. Chest. 2014;145(4):833-8.

62. Mitchell PD, Kennedy MP. Bronchoscopic management of malignant airway obstruction. Adv Ther. 2014;31(5):512-38.
63. Harms W, Krempien R, Grehn C, et al. Electromagnetically navigated brachytherapy as a new treatment option for peripheral pulmonary tumors. Strahlenther Onkol. 2006;182(2):108-11.

64. Becker HD, Harms W, Debus J, Bruekner D, McLemore T. Brachytherapy of inoperable peripheral lung cancer guided by electromagnetic navigation and endobronchial ultrasound: feasibility study and confirmation by long-term results at two centers. Chest Meet Abstr 2009;136(4). doi:10.1378/chest. 136.4_MeetingAbstracts.2S-f

65. Tsushima Y, Endo K. Analysis models to assess cost effectiveness of the four strategies for the work-up of solitary pulmonary nodules. Med Sci Monit. 2004;10(5):MT65-72.

66. Steinfort DP, Liew D, Irving LB. Radial probe EBUS versus CT-guided needle biopsy for evaluation of peripheral pulmonary lesions: an economic analysis. Eur Respir J. 2013;41(3):539-47.

67. Dale CR, Madtes DK, Fan VS, et al. Navigational bronchoscopy with biopsy versus computed tomography-guided biopsy for the diagnosis of a solitary pulmonary nodule: a cost-consequences analysis. J Bronchol Interv Pulmonol. 2012;19(4): 294-303.

68. Wada H, Hirohashi K, Nakajima T, et al. Assessment of the new thin convex probe endobronchial ultrasound bronchoscope and the dedicated aspiration needle: a preliminary study in the porcine lung. J Bronchol Interv Pulmonol. 2015;22(1):20-7. 